\title{
The Axial Double Probe and Fields Signal Processing for the MMS Mission
}

\author{
R.E. Ergun · S. Tucker · J. Westfall · K.A. Goodrich • D.M. Malaspina · D. Summers • \\ J. Wallace - M. Karlsson · J. Mack • N. Brennan - B. Pyke · P. Withnell • R. Torbert • \\ J. Macri · D. Rau · I. Dors · J. Needell · P.-A. Lindqvist · G. Olsson · C.M. Cully
}

Received: 8 May 2014 / Accepted: 14 October 2014 / Published online: 2 December 2014

(C) The Author(s) 2014. This article is published with open access at Springerlink.com

\begin{abstract}
The Axial Double Probe (ADP) instrument measures the DC to $\sim 100 \mathrm{kHz}$ electric field along the spin axis of the Magnetospheric Multiscale (MMS) spacecraft (Burch et al., Space Sci. Rev., 2014, this issue), completing the vector electric field when combined with the spin plane double probes (SDP) (Torbert et al., Space Sci. Rev., 2014, this issue, Lindqvist et al., Space Sci. Rev., 2014, this issue). Two cylindrical sensors are separated by over $30 \mathrm{~m}$ tip-to-tip, the longest baseline on an axial DC electric field ever attempted in space. The ADP on each of the spacecraft consists of two identical, $12.67 \mathrm{~m}$ graphite coilable booms with second, smaller $2.25 \mathrm{~m}$ booms mounted on their ends. A significant effort was carried out to assure that the potential field of the MMS spacecraft acts equally on the two sensors and that photo- and secondary electron currents do not vary over the spacecraft spin. The ADP on MMS is expected to measure DC electric field with a precision of $\sim 1 \mathrm{mV} / \mathrm{m}$, a resolution of $\sim 25 \mu \mathrm{V} / \mathrm{m}$, and a range of $\sim \pm 1 \mathrm{~V} / \mathrm{m}$ in most of the plasma environments MMS will encounter. The Digital Signal Processing (DSP) units on the MMS spacecraft are designed to perform analog conditioning, analog-to-digital (A/D) conversion, and digital processing on the ADP, SDP, and search coil magnetometer (SCM) (Le Contel et al., Space Sci. Rev., 2014, this issue) signals. The DSP units include digital filters, spectral processing, a high-speed burst memory, a solitary structure detector, and data compression.
\end{abstract}

R.E. Ergun $(\bigotimes) \cdot$ S. Tucker · J. Westfall $\cdot$ K.A. Goodrich · D.M. Malaspina · D. Summers · J. Wallace M. Karlsson · J. Mack · N. Brennan · B. Pyke · P. Withnell

Laboratory of Atmospheric and Space Physics, University of Colorado, Boulder, CO, USA

e-mail: ree@lasp.colorado.edu

R.E. Ergun · K.A. Goodrich

Department of Astrophysical and Planetary Sciences, University of Colorado, Boulder, CO, USA

R. Torbert · J. Macri · D. Rau · I. Dors · J. Needell

University of New Hampshire, Durham, NH, USA

P.-A. Lindqvist · G. Olsson

Royal Institute of Technology, Stockholm, Sweden

C.M. Cully

University of Calgary, Calgary, Canada 
The DSP uses precision analog processing with, in most cases, $>100 \mathrm{~dB}$ in dynamic range, better that $-80 \mathrm{~dB}$ common mode rejection in electric field $(E)$ signal processing, and better that $-80 \mathrm{~dB}$ cross talk between the $E$ and $\mathrm{SCM}(B)$ signals. The $\mathrm{A} / \mathrm{D}$ conversion is at 16 bits with $\sim 1 / 4$ LSB accuracy and $\sim 1$ LSB noise. The digital signal processing is powerful and highly flexible allowing for maximum scientific return under a limited telemetry volume. The ADP and DSP are described in this article.

Keywords Magnetospheric multiscale mission · Electric field instrument · Axial double probe $\cdot$ Digital signal processing

\section{Introduction}

The ADP measures the electric field component along the spin axis of the MMS spacecraft. It uses the double probe technique by sensing the local plasma potential with two cylindrical probes separated by $\sim 30 \mathrm{~m}$ tip-to-tip. The axial direction, which completes the vector electric field when combined with the spin-plane vector, is the most challenging component of the vector DC electric field measurement. The physical antenna lengths are constrained by mechanical limitations, which include deployment of stiff booms while preserving spacecraft stability.

The ADP (Fig. 1) on each of the four MMS spacecraft consists of two identical, $12.67 \mathrm{~m}$ graphite coilable booms (made by ATK Aerospace Systems). A second, smaller boom called the receiving element (RE) is mounted on the out-board end of each coilable boom (Fig. 2). These $2.25 \mathrm{~m}$ booms are folded on the top and bottom of the spacecraft for launch (Fig. 3). The deployment sequence has the RE released first, followed by the deployment of the $12.67 \mathrm{~m}$ coilable boom.

The design of the ADP involved significant consideration of the photo- and secondary electron currents to the sensors. One concern is variation of photoelectron currents with spacecraft spin. All elements of the 2.25-m REs are cylindrical after deployment, including the hinges. The stubs, preamplifiers and sensors are coated with graphite-epoxy (DAG 213) to assure consistent surface properties as the spacecraft rotates. The booms are helically twisted $60^{\circ}$ every two meters so that the illuminated area remains constant while rotating.

Equally important is the symmetry between the top $(+Z)$ and bottom $(-Z)$ sensors. The ADP booms are mounted to be symmetric about the spacecraft's electrostatic plane, which is dominated by the spin-plane wire booms. The SDP wire booms are offset to the $+Z$ side of the spacecraft center, so the $-\mathrm{Z}$ boom (underside of the spacecraft) is recessed into the spacecraft by $10 \mathrm{~cm}$ to maintain symmetry about the spacecraft's electrostatic plane. On orbit, the MMS spacecraft are to be oriented so that the ADP booms are within $5^{\circ}$ of normal to the sun. A guard ring, $30.9 \mathrm{~cm}$ in diameter and $2.6 \mathrm{~cm}$ high, encircles the tip plate at the end of the coilable boom to shadow the top surface. The guard ring is designed so that in the nominal attitude neither tip plate is exposed to the sun, promoting a nearly identical photoelectron environment between the two opposing sensors.

The ADP sensors are fed a bias current to optimize (reduce) the resistive coupling between the plasma and the sensor. To further control the photoelectron environment, the surface potentials of (a) the under side of the tip plate (called inner guard), (b) the topside of the tip plate and guard ring (called outer guard), and (c) the hinges, stubs, and preamplifier housing (called stub) are controlled. Each surface section can be set to $\pm 10 \mathrm{~V}$ with respect to the sensor's DC potential.

The ADP on MMS is expected to measure DC electric field with a precision of $\sim 1 \mathrm{mV} / \mathrm{m}$, a resolution of $\sim 25 \mu \mathrm{V} / \mathrm{m}$, and a range of $\sim \pm 1 \mathrm{~V} / \mathrm{m}$ in most of the plasma 
environments MMS will encounter. Constant offsets between the booms will be removed by two methods including minimizing $E \cdot B$ as measured by the double probe over long ( $>20 \mathrm{~s}$ ) periods and by comparison with the electric field as measured by the electron drift instrument (Torbert et al. 2014, this issue). The spectral power density of the ADP has a dynamic range is from $8 \times 10^{-15}(\mathrm{~V} / \mathrm{m})^{2} / \mathrm{Hz}$ to $10^{-3}(\mathrm{~V} / \mathrm{m})^{2} / \mathrm{Hz}$ at $10 \mathrm{kHz}$.

The DSP design is derived from that on the THEMIS spacecraft (Cully et al. 2007) with modifications that enhance the frequency response, for example, the sample rates are significantly higher. This unit receives nine signals (six voltage signals from the SDP and ADP and three signals from the SCM). It creates the electric field signals in analog, performs analog signal conditioning, the analog to digital conversion, and digital signal processing on the scientific signals. The digital signal processing includes digital filtering, spectral processing, burst memory, solitary wave detection, and data compression inside of a field-programmable gate array (FPGA). It also creates calibration signals for the SCM.

\section{Science Objectives and Requirements}

The MMS science objectives and requirements are discussed in companion articles (Burch et al. 2014, this issue; Torbert et al. 2014, this issue; Hesse et al. 2014, this issue), so we only provide a brief summary specific to the ADP and DSP.

\subsection{Science Objectives}

The primary science objectives of MMS focus on the ion and electron diffusion regions of magnetic reconnection. In these regions, the DC electric fields are expected to deviate from ideal magnetohydrodynamics (Hesse et al. 2014, this issue). The electron diffusion region and its separatrix are expected to have a number of possible plasma waves and plasma structures including Alfven waves, whistler waves, electron phase-space holes, and parallel electric fields (Drake et al. 2003; Lapenta et al. 2011). The diffusion region electric fields, $\mathrm{AC}$ magnetic fields, plasma waves, and plasma structures motivate the requirements on the ADP and DSP.

\subsection{Science Requirements}

The sensitivity and amplitude range of the DC electric fields, wave electric fields, and wave magnetic fields for MMS (Table 1) are based those predicted by magnetic reconnection theory (Burch et al. 2014, this issue; Burch and Drake 2009) and those recorded by previous missions in orbits similar to that of MMS. Plasma simulations predict that DC electric fields are expected to deviate from ideal behavior $(\boldsymbol{E}+\boldsymbol{u} \times \boldsymbol{B}=0$, where $\mathbf{E}$ is the electric field, $\mathbf{u}$ is the plasma flow velocity, and $\mathbf{B}$ is the magnetic field) from $\sim 1 \mathrm{mV} / \mathrm{m}$ to $\sim 10 \mathrm{mV} / \mathrm{m}$ in regions of magnetic reconnection (Hesse et al. 2014, this issue). This deviation is important for our understanding of the magnetic reconnection process and motivates the $\sim 1 \mathrm{mV} / \mathrm{m}$ accuracy. In previous missions, such accuracy has been achieved with long wire booms, but not routinely in the axial component of $\mathbf{E}$. The MMS ADP is designed to achieve such accuracy in most of the plasma environments explored by MMS. The expected amplitude range of the DC electric field is based on previous experience. The THEMIS mission observed fields as high as $500 \mathrm{mV} / \mathrm{m}$ (Ergun et al. 2009; Andersson et al. 2009), which provides the basis of the DC range on MMS.

The time scales that the plasma exhibits in regions of magnetic reconnection determine the frequency range of MMS electric and magnetic fields. In sub-solar reconnection regions 
Table 1 Primary requirements for ADP and DSP

\begin{tabular}{lllllll}
\hline Frequency range & $\begin{array}{l}\text { DC precision } \\
(\mathrm{mV} / \mathrm{m})\end{array}$ & $\begin{array}{l}\text { DC range } \\
\text { amplitude } \\
(\mathrm{mV} / \mathrm{m})\end{array}$ & $\begin{array}{l}\mathrm{AC} \\
\text { sensitivity }\end{array}$ & $\begin{array}{l}\text { AC } \\
\text { amplitude } \\
\text { range }\end{array}$ & $\begin{array}{l}\text { AC sensitivity/ } \\
\text { Amplitude range } \\
\text { units }\end{array}$ \\
\hline $\mathrm{SDP}$ & $\mathrm{DC}-100 \mathrm{kHz}$ & 0.5 & \pm 625 & $9 \times 10^{-16}$ & $10^{-4}$ & $(\mathrm{~V} / \mathrm{m})^{2} / \mathrm{Hz}$ at $10 \mathrm{kHz}$ \\
$\mathrm{ADP}$ & $\mathrm{DC}-100 \mathrm{kHz}$ & 1 & \pm 800 & $4 \times 10^{-14}$ & $10^{-3}$ & $(\mathrm{~V} / \mathrm{m})^{2} / \mathrm{Hz}$ at $10 \mathrm{kHz}$ \\
$\mathrm{SCM}$ & $2 \mathrm{~Hz}-10 \mathrm{kHz}$ & & & $4 \times 10^{-10}$ & $10^{2}$ & $\mathrm{nT}^{2} / \mathrm{Hz}$ at $1 \mathrm{kHz}$ \\
\hline
\end{tabular}

Table 2 Summary of mass and lengths of the ADP; Mass and power of the DSP

\begin{tabular}{|c|c|c|c|c|c|c|c|}
\hline \multicolumn{4}{|l|}{ ADP } & \multicolumn{2}{|c|}{ Receiving element } & \multicolumn{2}{|l|}{ DSP } \\
\hline Component & $\begin{array}{l}\text { Mass } \\
(\mathrm{kg})\end{array}$ & Component & $\begin{array}{l}\text { Length } \\
\text { (m) }\end{array}$ & Component & $\begin{array}{l}\text { Mass } \\
(\mathrm{kg})\end{array}$ & Component & $\begin{array}{l}\text { Mass } \\
(\mathrm{kg})\end{array}$ \\
\hline $\begin{array}{l}\text { Receiving } \\
\text { element }\end{array}$ & 0.42 & $\begin{array}{l}+\mathrm{Z} \text { base to } \\
\mathrm{S} / \mathrm{C} \text { center }\end{array}$ & 0.33 & Guard & 0.190 & Side A & 0.369 \\
\hline $\begin{array}{l}\text { Extensible } \\
\text { boom }\end{array}$ & 1.52 & $\begin{array}{l}-\mathrm{Z} \text { base to } \\
\mathrm{S} / \mathrm{C} \text { center }\end{array}$ & 0.23 & Hinges (2) & 0.097 & Side B & 0.360 \\
\hline $\begin{array}{l}\text { Launch } \\
\text { latches (2) }\end{array}$ & 1.25 & $\begin{array}{l}\text { Extensible } \\
\text { boom }\end{array}$ & 12.67 & Stubs (both) & 0.046 & Component & Power (W) \\
\hline $\begin{array}{l}\text { Boom } \\
\text { canister }\end{array}$ & 4.06 & $\begin{array}{l}\text { Receiving } \\
\text { element }\end{array}$ & 2.25 & Pre-amplifier & 0.060 & $\begin{array}{l}\text { One side } \\
\text { operating }\end{array}$ & 2.55 \\
\hline $\begin{array}{l}\text { Overall } \\
\text { (each) }\end{array}$ & 7.24 & $\begin{array}{l}\text { Overall } \\
\text { (tip-to-tip) }\end{array}$ & 30.40 & Sensor & 0.022 & & \\
\hline Component & MOI $\left(\mathrm{kg} \mathrm{m}^{2}\right)$ & & & & & & \\
\hline ADP (each) & 188 & & & & & & \\
\hline
\end{tabular}

the electron skin depth is $\sim 1 \mathrm{~km}$ and the motion of the magnetopause is often between $\sim 10 \mathrm{~km} / \mathrm{s}$ and $\sim 100 \mathrm{~km} / \mathrm{s}$. The electron diffusion region crossing time is expected to be $\sim 0.1 \mathrm{~s}$. This time scale is the basis of "survey data". Such data is required continuously for electric and magnetic field measurements so that these regions can be identified. The electron skin depth in the magnetotail is significantly larger, so the time scales are longer.

Plasma waves and structures can reach the plasma frequency, which can be as high as $100 \mathrm{kHz}$ in the sub-solar region. Waveforms up to these frequencies are required to fully analyze the electron diffusion regions. The time scales from these waveforms are the basis of the burst data (Fusilier et al. 2014, this issue; Baker et al. 2014, this issue). The plasma frequency in the magnetotail is often less than $\sim 10 \mathrm{kHz}$, so less time resolution is required.

\subsection{Mass, Power, Telemetry, and Moment of Inertia Constraints}

Space flight instruments are almost always heavily constrained by mass, power, telemetry, and cost. These constraints often dictate a significant part of the instrument design. The ADP has an additional constraint, the moment of inertia (MOI) that it imparts on the spacecraft. The MOI constraint has been the primary limiting factor in axial boom length on a number of previous missions (Pankow et al. 2001; Ergun et al. 2001; Bonnell et al. 2008). Shorter axial booms are one of the principal reasons that DC electric fields are less accurate in the axial component. A summary of the mass, power, and size of the ADP and DSP are listed in Table 2. 


\section{Instrument Description}

The ADP and DSP are an integral part of the MMS double probe electric field instrument. They combine with the SDP, boom electronics board (BEB), axial boom electronics board (AEB), and the central data processing unit as discussed in Torbert et al. (2014, this issue). The SDP and ADP sensors and preamplifiers produce six voltage signals from $-100 \mathrm{~V}$ to $+40 \mathrm{~V}$. The BEB and AEB provide floating power to the preamplifiers and control the bias currents, guard, and stub voltages at the sensors as well (Lindqvist et al. 2014, this issue). The six voltage signals and three SCM signals are routed to the DSP. The DSP derives electric field signals from the voltage signals and processes all of the signals for transmission. Ultimately, the DSP is designed to optimize the scientific return under the limited data volume due to telemetry restrictions.

\subsection{Axial Double Probe}

The ADP (Fig. 1) is comprised of two booms deployed along the spin axis of each MMS spacecraft, one boom in the $+\mathrm{Z}$ direction and one in the $-\mathrm{Z}$ direction. At the tip of each $12.67 \mathrm{~m}$ long extensible boom is a $2.25 \mathrm{~m}$ long receiving element. Fully deployed, the tipto-tip distance between the two ADPs is 30.4 meters.

The ADP booms and REs are designed to have cylindrical symmetry, primarily to eliminate modulation of the photoelectron current as the spacecraft rotates. The design also optimizes symmetry between the $+\mathrm{Z}$ and $-\mathrm{Z}$ sensors; opposing sensors experience nearly the same potential created by the spacecraft (and its booms) and they have nearly identical photo- and secondary electron environments. The design process included a detailed estimate of the electrostatic center of the spacecraft so the sensors are nearly equidistant from the electrostatic center of the spacecraft (see Cully et al. 2007). The MMS spacecraft are required to maintain attitude so that the principle body axis of the spacecraft is within $5^{\circ}$ of normal to the spacecraft-sun line. Guard rings were placed on the ends of the extensible booms so that the surfaces of the tip plates facing the sensors have no photoelectron emission with solar incidence angles up to $5^{\circ}$ from normal to the RE. The careful implementation of symmetry and the long baseline of the ADP distinguish the MMS electric field instrument from previous efforts.

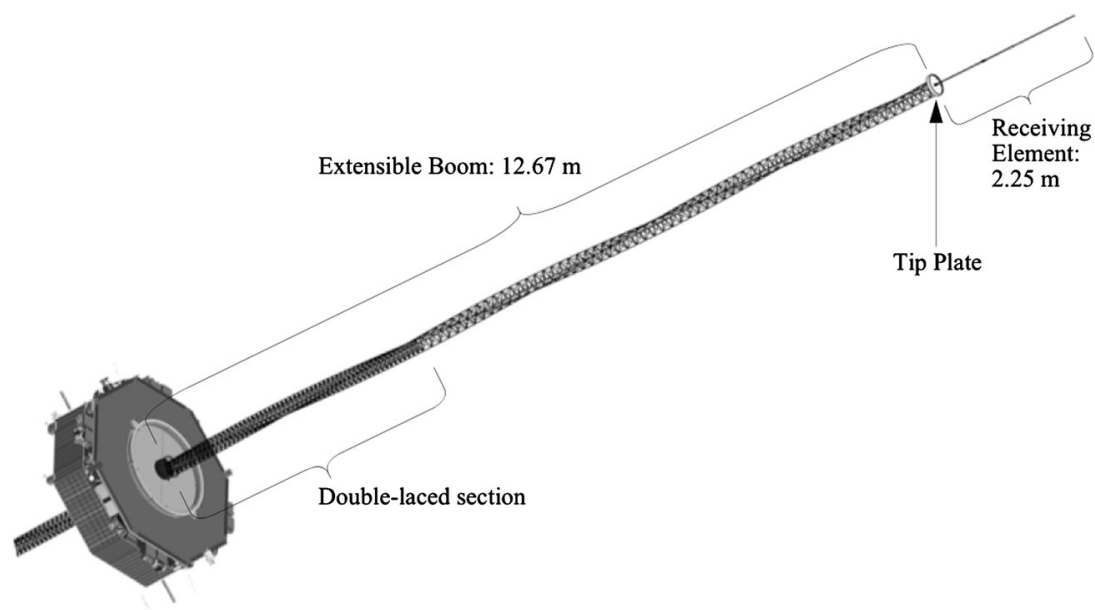

Fig. 1 The ADP boom and RE deployed from the MMS spacecraft. Figure 2 details the RE 


\subsubsection{ADP Extensible Booms}

The ADP extensible boom (Fig. 1) was developed by ATK Aerospace Systems. The trusslike boom structure consists of three monolithic graphite composite longerons that run the full length of the boom. The longerons are held in place by graphite composite battens (providing separation force between longerons) and non-magnetic stainless steel wire rope diagonals (limiting the separation between longerons). When deployed, the battens are compressed and the diagonals are under tension. The resulting tensional integrity structure is a stiff beam-like truss with no measurable mechanical dead band. The lower section of the boom (Fig. 1) is "double-laced" with twice the number of battens and diagonals so the buckling strength of the boom is increased when subjected to a transverse load. The total deployment stroke of the boom tip is 12.41 meters while the total length from root to tip is 12.67 meters. The triangular boom cross-section has a maximum diameter of $26.5 \mathrm{~cm}$. The boom is helically twisted $60^{\circ}$ every two meters so that the illuminated area remains constant while rotating. This geometry limits the modulation of photoelectron generation near the ADP RE. Nearly all of the exterior surfaces of the boom are conductive. The boom structure is electrically isolated from the MMS spacecraft chassis and is electrically connected to the DSP analog ground. A shielded cable with five conductors runs the length of each of the boom's longerons, which allows critical signals to be wired redundantly. Signals are routed through a breakout board at the tip plate, which connects to the ADP Receiving Element.

The ADP booms and RE are designed to have symmetry between the $-\mathrm{Z}$ sensor and the $+Z$ sensor. In order to compensate for the fact that the SDP booms are physically positioned near the $+Z$ surface the $-\mathrm{Z}$ ADP is recessed $10 \mathrm{~cm}$ into the spacecraft relative to the $+Z$ ADP. The magnetometer booms are deployed from the $-Z$ deck, but have far less impact on the electrostatic center than does the SDP. Under this arrangement, the two RE sensors are nearly equidistant from the predicted electrostatic center of the spacecraft, which decreases the unwanted effect of the spacecraft potential in the axial electric field measurement.

\subsubsection{ADP Receiving Elements}

Fig. 2 displays a close-up view of the RE, which mounts to the tip of the extensible boom. A disc-shaped tip plate covers the end of the boom. The two sides of the tip plate are electrically isolated from each other as well as from the ADP Boom. The surface facing the boom and the spacecraft is called the inner guard. The outer surface, called the outer guard, faces the RE. The inner and outer guard potentials are controlled by the AEB. The outer guard has a shadow ring, $30.9 \mathrm{~cm}$ in diameter and $2.6 \mathrm{~cm}$ high, which encircles a the tip plate at the end of the coilable boom. The guard ring is electrically connected to the outer guard. It shadows sunlight from the tip plate surface for solar incidence angles up to $5^{\circ}$ from normal to the RE. This geometry prevents photoemission on surfaces that face the ADP sensor with the exception of the top edge of the guard ring, preserving symmetry in the photoelectron environment between the $+\mathrm{Z}$ and $-\mathrm{Z}$ sensors.

When deployed, the REs are comprised of (going away from the spacecraft) a $90^{\circ}$ base hinge, a $0.78 \mathrm{~m}$ long by $1 \mathrm{~cm}$ diameter tube, a $180^{\circ}$ hinge, a $0.43 \mathrm{~m}$ long by $0.95 \mathrm{~cm}$ diameter tube, a $5.6 \mathrm{~cm}$ long by $2.1 \mathrm{~cm}$ diameter preamplifier, and a $1 \mathrm{~m}$ long by $0.64 \mathrm{~cm}$ diameter sensor. The exposed surface of the base hinge and all of the RE up through the preamplifier are controlled by the AEB as the "stub potential". In total length of the "stub section" is 1.25 meters long. The tubes are coated with DAG213 (graphite dispersed in an epoxy matrix). The hinges, which are cylindrically symmetric once deployed, are plated with electroless nickel with co-deposited PTFE (polytetrafluoroethylene), which is a non-conductive, low-fiction material. The total area of PTFE that is exposed to the plasma is much less that 


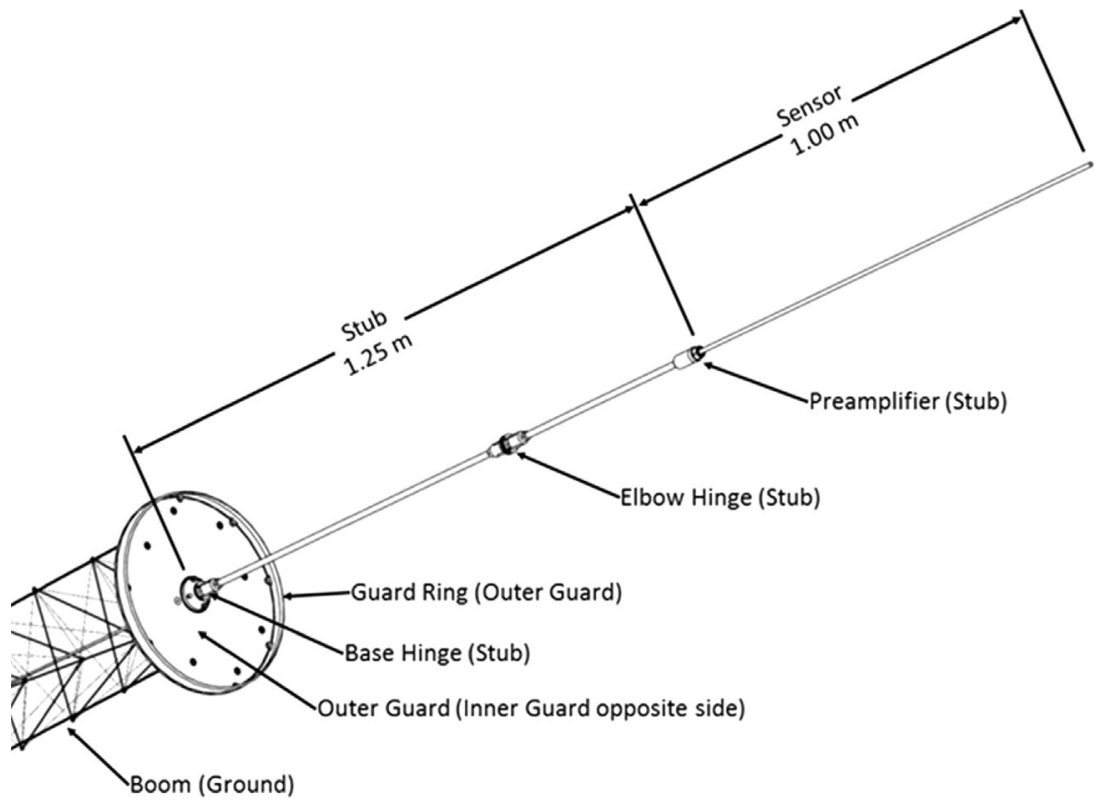

Fig. 2 The receiving element at the end of the extensible boom. The potentials of all of the surfaces near the sensor are controlled by the AEB. The boom is at ground. The inner guard (not in drawing) faces the boom and spacecraft. The outer guard is the ring and the surface at the end of the boom that faces the receiving element. The hinges, stub elements, and preamplifier case are held at the "stub" potential

of the electroless nickel, so no significant impact from PTFE charging is expected. The base hinge rotates $90^{\circ}$ and the elbow hinges rotate $180^{\circ}$. The ADP preamplifier resides at the end of the stub and inboard of the sensor. Electrical connection to the preamp is made via a 1.25-meter flex circuit, which is routed inside the stub and through the hinge bodies thus maintaining the axial symmetry of the stub section. The ADP preamplifier housing is 5.6 $\mathrm{cm}$ long and $2.1 \mathrm{~cm}$ in diameter. The outer shell of the preamp housing is electrically connected to the stub and coated with DAG213. The preamp electronics are encased in a brass radiation shield (not exposed to the plasma), which is electrically connected via a resistor to preamp output. This electrical "boot strapping" reduces capacitive coupling with the preamp case and increases high-frequency ( $>1 \mathrm{kHz}$ ) performance. The 1.0 -meter long by $0.64 \mathrm{~cm}$ diameter cylindrical sensor tube is mounted to outboard end of the preamp and electrically connected to the preamp input. Bias current to the sensor is provided by the AEB.

The floating power supplies for the ADP preamplifiers are at $\pm \sim 14 \mathrm{~V}$ from the "floating ground". The floating ground is driven at the DC $-\sim 300 \mathrm{~Hz}$ output of each of the preamplifiers. These electronics are located on the AEB. The floating ground has a minimum range from $-100 \mathrm{~V}$ to $+40 \mathrm{~V}$. Previous missions (e. g. Polar and Cluster II) have shown that the spacecraft may charge to large positive potentials (up to nearly $100 \mathrm{~V}$ ) whereas negative potentials are rare and significantly less (except in radiation belts). The floating ground range was chosen so that the ADP can operate over the range of expected potentials for the MMS spacecraft in regions of magnetic reconnection.

\subsubsection{ADP Deployment}

The stowed extensible booms and RE are displayed in Fig. 3. The ADP extensible boom is coiled inside of a canister, and the RE is folded and held in place by two launch latch 


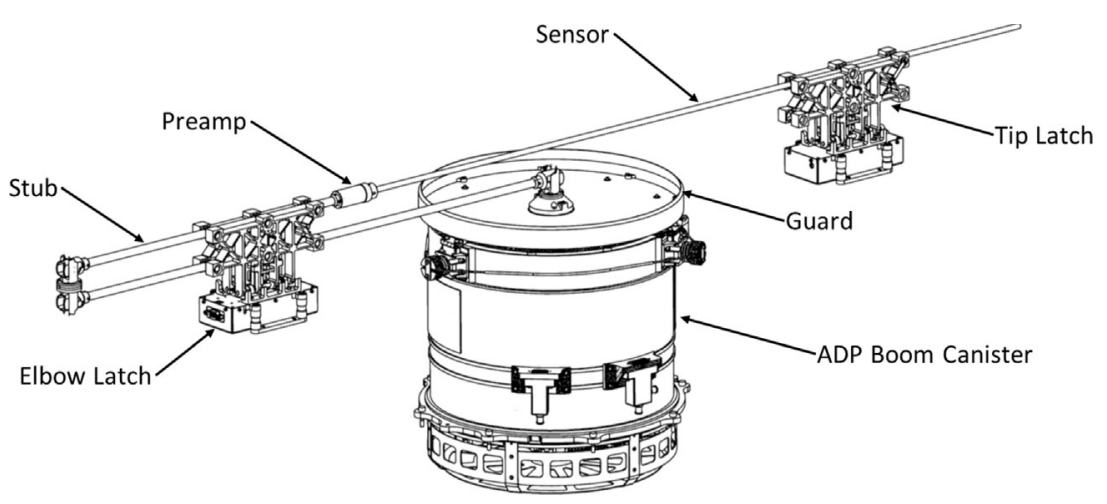

Fig. 3 The ADP in the stowed configuration

release mechanisms. The RE folds $90^{\circ}$ at its base and $180^{\circ}$ at its elbow. The $180^{\circ}$ elbow hinge is comprised of two $90^{\circ}$ hinges placed back-to-back. The launch latches clamp the stub and sensor tubes between two spring-loaded latch arms. At each latch, three staticdissipative acetal pads contact the sensor and stub. A high-output paraffin actuator (HOP, Sierra Nevada Corporation) opens the latches. The HOP actuator pushes the release mechanism over-center, which results in a near simultaneous release of the latch arms in a single latch. The tip latch is released first, and the elbow latch is released second. Since one latch is sufficient to constrain the RE, the RE begins deploying when the elbow latch is released. Redundant torsion springs in each hinge provide the strain energy for RE deployment. Each hinge contains an internal lockout mechanism that prevents the RE from rebounding more than $5^{\circ}$. Fully deployed, the hinge springs hold the hinge against a hard stop with enough torque to prevent the hinge from flexing during routine attitude control maneuvers.

The ADP boom is coiled inside of $29 \mathrm{~cm}$ diameter by $22 \mathrm{~cm}$ high canister. Before and during launch, the boom is secured within the canister by three spring-loaded pins, which hold the tip plate in place on top of the canister. Deployment is by a Frangibolt actuated release mechanism (TiNi Aerospace Corporation). A TiNi shape memory actuator breaks the Frangibolt, which releases a tensioned cable that secures the three spring-loaded pins. After the pins pull back, the boom tip is allowed to move freely from the canister. The initial motion is generated by three kick-over springs located at the base of the boom. These springs rotate and erect the lowest portion of the three longerons into the deployed position, which pushes the coiled stack out of the canister. The boom then uncoils and deploys via strain energy in the longerons from the base to the tip. The rate of deployment is controlled by a damper mechanism that reels out a lanyard attached to the tip as the boom deploys. A camactuated microswitch on the damper mechanism measures deployed length. Depending on thermal conditions, the ADP Boom deployment takes approximately four to five minutes.

Each boom and RE is deployed separately. The planned sequence has the REs deployed first, prior to the SDP and magnetometer boom deployment. The ADP booms are fully deployed after the magnetometer booms and SDP wire booms. The MMS spacecraft $(\sim 1000 \mathrm{~kg}$ ) have sufficient moment of inertia so that they are spin-stable with the ADP's fully deployed without the SDP wire booms deployed.

\subsubsection{ADP Boom Dynamics, Alignment, and Stability}

The first bending mode of vibration of a fully deployed ADP is measured at $0.53 \mathrm{~Hz}$. This frequency is sufficient to isolate the ADP from the harmonics that result from repeated radial 
thruster firing during spacecraft maneuvers. During maneuvers, thrusters are fired twice per spin creating a periodic-pulse profile of acceleration versus time. The thruster excitations are at harmonics of $0.1 \mathrm{~Hz}$ under the nominal 20-second spin period. Because of the uncertainty in the on-orbit first bending mode frequency of ADP as well as uncertainty in its damping behavior in the space environment, the ADP is designed with sufficient strength to withstand acceleration with harmonics near its predicted first bending mode frequency during extended periods of radial thruster firing.

Alignment of the ADP is critical to maintaining a shadow on the outer guard of the $\mathrm{RE}$ during active science operations. The alignment can also impact the mass properties of the spacecraft. Due to its length, ADP misalignment could result in significant error in the principal spin axis relative the geometric axis of the spacecraft. For example, a $1^{\circ}$ misalignment of the ADP could result in a 3.6 $6^{\circ}$ offset of the spacecraft spin axis. A spin axis error affects the pointing of ADP as well as other MMS instruments. The ADP misalignment from all sources (boom alignment, spacecraft interface alignment, and thermal distortions) is constrained to be under $0.18^{\circ}$. If a $0.18^{\circ}$ misalignment is realized in both ADP booms in opposite directions, the principal spin axis can be offset by as much as $0.65^{\circ}$ from the geometric Z-axis. Such a misalignment is scientifically acceptable and is stable.

A major consideration in the ADP design is a moment of inertia (MOI) constraint. The moment of inertia about the spin axis $\left(I_{z z}\right)$ must be greater that the largest moment of inertia of any axis in the spin plane $\left(I_{r r}\right)$ in order to maintain spin stability. Prior to deployment of the ADP booms, the difference between $I_{z z}$ and $I_{r r}$ of the disk-shaped MMS spacecraft is calculated to be $590 \mathrm{~kg} \mathrm{~m}^{2}$ not including the SDP wire boom inertial effects and fuel. The ADPs thus are constrained to add less than $200 \mathrm{~kg} \mathrm{~m}^{2}$ each to $I_{r r}$ after deployment to maintain a spacecraft spin stability ratio $\left(I_{z z}\right.$ to $\left.I_{r r}\right)$ of 1.15 . This MOI constraint leads to a design with the smallest possible mass at the outer end of the ADP. This minimization was achieved through use of graphite composite longerons on the extensible boom and minimal structure on the RE tubes and hinges. The RE (420 gm.), for example, cannot be deployed in surface gravity nor can it withstand launch vibration without the support form the launch latches. The 30.4-meter length is primarily limited by the MOI constraint.

\subsection{Digital Signal Processor}

The DSP (Fig. 4) receives nine analog signals from the ADP, SDP, and SCM sensors. It performs analog signal conditioning, A/D conversion, and digital signal processing using a FPGA (Actel Corporation RTAX2000). The DSP also creates calibration signals for the SCM.

There are two DSPs on each of the MMS spacecraft. One is active while the other is an unpowered spare. The units are identical except for the signal connections and minor grounding differences. DSP A receives the analog signals and routes them directly to DSP B. The analog inputs are high impedance on each of the DSP units, so the powered-off unit does not disturb the sensor signals. The BEB, AEB, and SCM grounds are connected through DSP A directly while both DSP A and DSP B are connected to analog ground on a common backplane. There are no significant differences in performance between the two units. More information on the redundant operation of the FIELDS suit is in Torbert et al. (2014, this issue).

\subsubsection{DSP Analog Conditioning}

The six analog signals from the ADP and SDP have required minimum amplitude range of $-100 \mathrm{~V}$ to $+40 \mathrm{~V}$ (BEB and AEB performances are discussed in Lindqvist et al. 


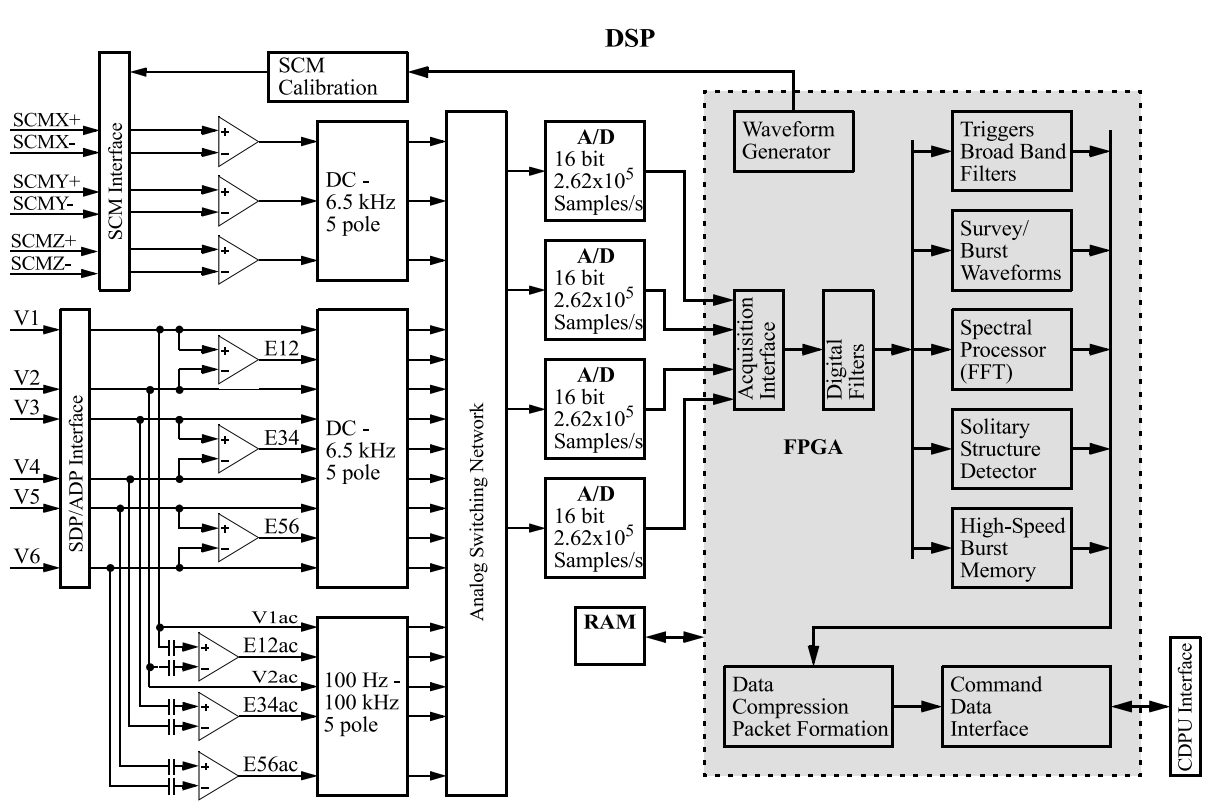

Fig. 4 A notional block diagram of the scientific signal flow in the DSP. V1 to V4 are from the SPD. V5 are V6 are ADP signals

2014 , this issue) in the frequency range from DC to $\sim 100 \mathrm{~Hz}$. These "V" signals are labeled V1 through V6. V1 and V2 are opposing spin-plane probes, as are V3 and V4. V5 and V6 are opposing ADP probes. The DSP can accept the ADP and SDP signals from $-120 \mathrm{~V}$ to $+120 \mathrm{~V}$. The high-frequency $(1 \mathrm{kHz}-100 \mathrm{kHz})$, undistorted amplitude range from the preamplifiers is expected to be $\pm 12.5 \mathrm{~V}$ from the DC level, so the DSP has a smaller dynamic range $( \pm 12.5 \mathrm{~V})$ on AC-coupled signals. The three SCM signals have an amplitude range of $\sim \pm 6.2 \mathrm{~V}$. The gains of all nine signals are fixed at the DSP input interface to avoid saturation during signal conditioning and once again prior to $\mathrm{A} / \mathrm{D}$ conversion to adjust to the range to the particular $\mathrm{A} / \mathrm{D}$ converter. The net gains, ranges, and sensitivities are called out in Table 3.

The DSP constructs electric field signals by taking the difference of the signals from opposing sensors. For example, E12 (DC-coupled) is generated from V1-V2. The common mode rejection ratio of the DC-coupled signals is designed to be $-80 \mathrm{~dB}$ or better using precision resistors so that the spacecraft potential does not influence the generation of electric field signals. The DSP also constructs AC-coupled signals with a one-pole high-pass filter at $100 \mathrm{~Hz}$. AC-coupled signals have higher gains in both Eac and Vac signals and thus higher sensitivity. The common mode rejection of the AC channels is $-60 \mathrm{~dB}$ in the pass band. The AC-coupled ranges match preamplifier performance.

The DC-coupled signals and the SCM signals are conditioned for A/D conversion with five-pole low-pass Bessel filters. The Bessel filters have a $-3 \mathrm{~dB}$ gain at $\sim 6.5 \mathrm{kHz}$, which is 0.8 of the Nyquist frequency or 0.4 of the sampling rate. The Bessel filters are designed to have a constant time delay in the pass band (linear phase delay) so that the time-domain signal shape is minimally distorted. This feature preserves the signature of plasma structures such as electron phase-space holes and double layers. The DC-coupled signals and the SCM signals are fed to a switching network (a set of analog switches controlled by the FPGA) that channels them into a single, 16-bit A/D converter. Each of the twelve signals is 
Table 3 Gains, ranges and sensitivities of ADP, SDP, and SCM signals

\begin{tabular}{|c|c|c|c|c|c|c|c|c|c|}
\hline \multirow[t]{3}{*}{ Signal(s) } & \multicolumn{2}{|c|}{ Antenna length ${ }^{1}$} & \multirow{3}{*}{$\begin{array}{l}\text { DSP } \\
\text { gain }^{2}\end{array}$} & \multicolumn{3}{|c|}{$\mathrm{DC}-100 \mathrm{~Hz}$} & \multicolumn{3}{|c|}{$\mathrm{AC}: \mathrm{At} \sim 5 \mathrm{kHz}$} \\
\hline & \multirow[t]{2}{*}{ Physical } & \multirow[t]{2}{*}{ Effective } & & \multirow{2}{*}{$\begin{array}{l}\text { Preamp } \\
\text { gain }\end{array}$} & \multicolumn{2}{|l|}{ Amplitude } & \multirow{2}{*}{$\begin{array}{l}\text { Preamp } \\
\text { gain }^{4}\end{array}$} & \multicolumn{2}{|l|}{ Amplitude } \\
\hline & & & & & Resolution & Range $^{3}$ & & Resolution & Range $^{5}$ \\
\hline V1-V4 & $60 \mathrm{~m}$ & $48 \mathrm{~m}$ & 0.0208 & 1.00 & $3.7 \mathrm{mV}$ & $-100-30 \mathrm{~V}$ & 0.85 & $4.4 \mathrm{mV}$ & $\pm 14.7 \mathrm{~V}$ \\
\hline V5, V6 & $15.1 \mathrm{~m}$ & $7.3 \mathrm{~m}$ & 0.0208 & 1.00 & $3.7 \mathrm{mV}$ & $-100-30 \mathrm{~V}$ & 0.8 & $4.6 \mathrm{mV}$ & $\pm 15.6 \mathrm{~V}$ \\
\hline $\begin{array}{l}\text { V1ac; } \\
\text { V2ac }\end{array}$ & $60 \mathrm{~m}$ & $48 \mathrm{~m}$ & 0.1991 & - & - & - & 0.85 & $9.4 \mu \mathrm{V} / \mathrm{m}$ & $\pm 306 \mathrm{mV} / \mathrm{m}$ \\
\hline E12; E34 & $120 \mathrm{~m}$ & $96 \mathrm{~m}$ & 0.0415 & 1.00 & $19 \mu \mathrm{V} / \mathrm{m}$ & $\pm 626 \mathrm{mV} / \mathrm{m}$ & 0.85 & $22 \mu \mathrm{V} / \mathrm{m}$ & $\pm 306 \mathrm{mV} / \mathrm{m}$ \\
\hline E56 & $30.4 \mathrm{~m}$ & $14.5 \mathrm{~m}$ & 0.211 & 1.00 & $25 \mu \mathrm{V} / \mathrm{m}$ & $\pm 817 \mathrm{mV} / \mathrm{m}$ & 0.8 & $31 \mu \mathrm{V} / \mathrm{m}$ & $\pm 1.02 \mathrm{~V} / \mathrm{m}$ \\
\hline $\begin{array}{l}\text { E12ac; } \\
\text { E34ac }\end{array}$ & $120 \mathrm{~m}$ & $96 \mathrm{~m}$ & 0.0996 & - & - & - & 0.85 & $9.3 \mu \mathrm{V} / \mathrm{m}$ & $\pm 306 \mathrm{mV} / \mathrm{m}$ \\
\hline E56ac & $30.4 \mathrm{~m}$ & $14.5 \mathrm{~m}$ & 0.1991 & - & - & - & 0.8 & $33 \mu \mathrm{V} / \mathrm{m}$ & $\pm 1.08 \mathrm{~V} / \mathrm{m}$ \\
\hline
\end{tabular}

${ }^{1}$ Effective antenna lengths in table are physical lengths times estimated shorting factors. More accurate effective antenna lengths are determined through on-orbit calibration

${ }^{2} \mathrm{DSP}$ gain is configured for A/D conversion range of $\pm 2.49 \mathrm{~V}$

${ }^{3}$ Preamp gain is frequency dependent. Gains in table are representative. See gain versus frequency charts

${ }^{4}$ Voltage ranges are minimum guaranteed ranges from preamplifier, BEB, and AEB. Actual ranges may be more extensive. Electric field ranges are limited by DSP A/D saturation

${ }^{5} \mathrm{AC}$ ranges are based on $\pm 12.5 \mathrm{~V}$ output from preamplifiers. Undistorted output depends on frequency and can be lower. See gain versus frequency charts. E56dc and E56ac ranges are limited by DSP A/D saturation

sampled at 16384 samples per second (S/s) with the A/D converter operating at $\sim 262 \mathrm{kS} / \mathrm{s}$ $\left(2^{18} \mathrm{~S} / \mathrm{s}\right)$. These signals are sampled sequentially every $\sim 3.8 \mu \mathrm{s}$. Sampling time differences are deterministic and included in the data timing (see Torbert et al. 2014, this issue).

There are nine DC-coupled E and V signals and three SCM signals using sixteen possible channels in the A/D converter leaving four empty channels. The nine $\mathrm{E}$ and $\mathrm{V}$ signals are sampled sequentially. Each of the SCM signals is given two sample periods for additional settling time. The remaining unused A/D channel is inserted between the SCM and electric field signals. The resulting isolation yields better than $-80 \mathrm{~dB}$ signal crosstalk between E and SCM signals. This strong isolation allows for accurate determination of E/B for wave mode identification and distinguishing electrostatic from electromagnetic features in magnetic reconnection and other plasma phenomena.

AC-coupled signals have a one-pole high-pass filter at $100 \mathrm{~Hz}$ and are given a higher gain on SDP signals. These signals are conditioned with a $100 \mathrm{kHz}$ Bessel filter $(-5 \mathrm{~dB})$. Three of the five AC-coupled signals can be selected for A/D conversion at $\sim 262 \mathrm{kS} / \mathrm{s}$ each. Three 16-bit A/D converters sample the AC-coupled signals simultaneously. The DSP has four identical A/D converters (Fig. 4).

\subsubsection{DSP Digital Processing}

The digital signal processing is designed to optimize the scientific return of the ADP, SDP, and SCM signals under the allocated data volume in the MMS telemetry system (Torbert et al. 2014, this issue; Fusilier et al. 2014, this issue). The raw signals from the ADP, SDP, and SCM could produce more than $10^{4}$ times the allocated data volume, so data selection and compression is critical to the success of the MMS mission. A primary part of the data selection occurs at the mission level, which includes all four observatories. All instruments 
on the MMS mission are designed to produce "survey" data, which are continuous, lowrate data throughout the orbit. Some instruments also produce burst data, which include the highest data rates and are selected on scientific interest. Only $\sim 2 \%$ to $\sim 3 \%$ of all burst data is expected to be transmitted and recorded for scientific analysis.

The DSP is designed to operate within the MMS mission system by producing both survey data and burst data. The DSP, however, must also further reduce the data volume. There are five primary scientific algorithms in the DSP including (1) broad band filters, (2) digital filtering of waveforms, (3) spectral processing, (4) burst memory, and (5) solitary structure detection. These algorithms are described below in addition to the SCM calibration, data compression, and other data handling functions.

Broadband Filters and Waveforms The raw data from the A/D converters is parsed and reformed into specific data types at the acquisition interface (Fig. 4). These reformed data are available to the scientific algorithms. The broadband filters and waveform processing start with a cascading finite impulse response (FIR) filter. The FIR filter convolves the input data stream $(x)$ with the function:

$$
y_{n}^{m-1}=a_{1} x_{n+3}^{m}+\cdots+a_{4} x_{n}^{m}+\cdots+a_{7} x_{n-3}^{m},
$$

where $\boldsymbol{a}=\{-8,0,72,128,72,0,-8\} / 256$. The integer $n$ represents the position in the time sequence and the integer $m$ (negative values of $0,-1,-2$, etc.) indicates the frequency band, $f_{m}=f_{o} 2^{m}$. The FIR coefficients themselves are values of $2^{i}$ (where $i$ is an integer) or a sum of two values of $2^{i}$, so execution of the FIR convolution is rapid and requires little resource within the FPGA. Most importantly, no multiplier is required. The resulting data stream, $y_{n}^{m-1}$, is filtered to a frequency band from DC to $f_{m-1}=f_{o} 2^{m-1}$ (see Cully et al. 2007 for frequency response of the FIR filters). The filtered signal, $y_{n}^{m-1}$, then takes two paths. It is passed through to a second FIR to create decimated low-pass waveform data (frequency band DC $\left.-f_{m-1}\right)$, and it is subtracted from the original data $\left(x_{n}^{m}\right)$, point by point, to create band-pass data for the broadband filters (frequency band of $f_{m-1}$ to $f_{m}$ ).

To create decimated waveform data $\left(x_{n}^{m-1}\right), y_{n}^{m-1}$ passes through a second FIR:

$$
z_{n}^{m-1}=0.25 y_{n+1}^{m-1}+0.5 y_{n}^{m-1}+0.25 y_{n-1}^{m-1} \text {. }
$$

$x_{n}^{m-1}$ is created from every other point of $z_{n}^{m-1}$. The resulting data $\left(x_{n}^{m-1}\right)$ has $1 / 2$ of the frequency range and possesses $1 / 2$ of the sample rate of the original $\left(x_{n}^{m}\right)$ data. This process is performed repeatedly, halving the frequency and sample rate with each iteration. Each iteration creates a new stream of waveform data and a new stream of band-pass data. The sample rates available from the cascading digital filters range in powers of two from as low as $1 \mathrm{~S} / \mathrm{s}$ to the native sampling rate $(16384 \mathrm{~S} / \mathrm{s}$ or $262144 \mathrm{~S} / \mathrm{s})$.

The frequency ranges of the band-pass data from the cascading digital filters are listed in Table 4. These data are further reduced to average amplitudes and/or peak amplitudes (configurable). The former averages the absolute value of the band-pass data over the reporting interval, nominally planned to be 10 seconds to work with the MMS burst system. These data are used as burst triggers and yield coarse spectral coverage at higher time resolution than the spectra created by fast Fourier transform (FFT).

Waveform data (except at the native rate) are processed through a FIR with a sevenelement kernel, which causes the filtered data to be delayed. To avoid timing errors, the delays are carefully calculated for each of the output taps. These delays are removed in the data processing software after transmission, which can recreate relative time between channels to less than $\sim 1 \mu \mathrm{s}$, and absolute time to $\sim 4 \mu \mathrm{s}$. Details of the timing are discussed in 
Table 4 Broadband filter bank frequency bins

\begin{tabular}{|c|c|c|c|c|c|c|c|}
\hline Bin & $\begin{array}{l}\text { Frequency range } \\
(\mathrm{Hz})\end{array}$ & Bin & $\begin{array}{l}\text { Frequency range } \\
(\mathrm{Hz})\end{array}$ & Bin & $\begin{array}{l}\text { Frequency range } \\
(\mathrm{Hz})\end{array}$ & Bin & $\begin{array}{l}\text { Frequency range } \\
(\mathrm{kHz})\end{array}$ \\
\hline \multicolumn{4}{|c|}{$16384 \mathrm{~S} / \mathrm{s}$ Native rate } & \multicolumn{4}{|c|}{$262144 \mathrm{~S} / \mathrm{s}$ Native rate } \\
\hline 0 & $0.8-1.5$ & 7 & $100-200$ & 0 & $25-50$ & 7 & $3.2-6.5$ \\
\hline 1 & $1.5-3$ & 8 & $200-400$ & 1 & $50-100$ & 8 & $6.5-13$ \\
\hline 2 & $3-6$ & 9 & $400-800$ & 2 & $100-200$ & 9 & $13-26$ \\
\hline 3 & $6-12$ & 10 & $800-1600$ & 3 & $200-400$ & 10 & $26-52$ \\
\hline 4 & $12-25$ & 11 & $1600-3200$ & 4 & $400-800$ & 11 & $52-104$ \\
\hline 5 & $25-50$ & 12 & $3200-6500$ & 5 & $800-1600$ & & \\
\hline 6 & $50-100$ & & & 6 & $1600-3200$ & & \\
\hline
\end{tabular}

Torbert et al. (2014, this issue). It is also possible to select any given waveform, for example E12, at multiple rates. This flexibility allows for simultaneous data flow for the survey stream and the burst stream at the same time.

Power Spectra The spectral processing on MMS is derived from to that used on THEMIS (Cully et al. 2008). Spectra are calculated via a 1024-point FFT algorithm on piecewise continuous sets of waveform data. Nine signals can be processed simultaneously. Six of the twelve DC-coupled E, DC-coupled V, or SCM signals (16384 S/s) are selected for spectral processing at $100 \%$ duty cycle. In addition, the three AC-coupled signals $(\sim 262 \mathrm{kS} / \mathrm{s})$ each can be processed at $6.25 \%$ duty cycle. Each of the nine signals has 16, 1024-point FFT operations every second; the FPGA performs 144 FFTs per second.

The FFT is performed by an arithmetic logic unit (ALU), which is controlled by a state machine. Both are hard-coded into the FPGA. The operation starts by applying a 1024-point Hanning window onto a waveform. Next, an FFT is implemented. The FFT is broken into a series of "butterfly" operations performed by the ALU. The result has real and imaginary data. Power spectra are calculated by taking the sum of squares of real and imaginary values (the ALU includes a multiplier), which produces a power spectrum with 512 frequency bins.

The frequency bins are then combined to give pseudo-logarithmic frequency spacing $(\delta f / f)$. The spectra are reduced to 56 frequency bins with $\delta f / f$ between $6 \%$ and $12 \%$ when possible. Narrow-band emissions can be fit to an accuracy of $\delta f / f \sim 3 \%$, allowing for an accurate determination of plasma density. Table 5 summarizes the frequency and amplitude ranges of the spectral processing.

The spectra can be averaged in time. The fastest reporting rate of any signal is 16 spectra per second. Reporting rates can be as slow a one spectra every $16 \mathrm{~s}$ (averaging 256 spectra). The averaging process has 48-bit accuracy to maximize the dynamic range. The amplitudes undergo a pseudo-logarithmic compression to an 8-bit number representing over $\sim 120 \mathrm{~dB}$ of dynamic range at $\sim 5 \%$ precision.

High-Speed Burst Memory The high-speed burst memory (HSBM) on the DSP is distinct from the burst memory of the central instrument data processing unit (Fusilier et al. 2014, this issue). It is designed to select the highest amplitude signals in AC-coupled waveforms and SCM waveforms. It accepts sample rates of AC-coupled waveforms from $\sim 16.4 \mathrm{kS} / \mathrm{s}$ to the native rate of $\sim 262 \mathrm{kS} / \mathrm{s}$. It receives only the native rate of the SCM signals $(\sim 16.4 \mathrm{kS} / \mathrm{s})$. Combined, these data have too high of a rate to be recorded continuously by the central MMS burst memory. The HSBM uses a $\sim 4.19$ Mbyte $\left(2^{22}\right.$ bytes) memory, 
Table 5 Summary of spectral processing and sensitivities

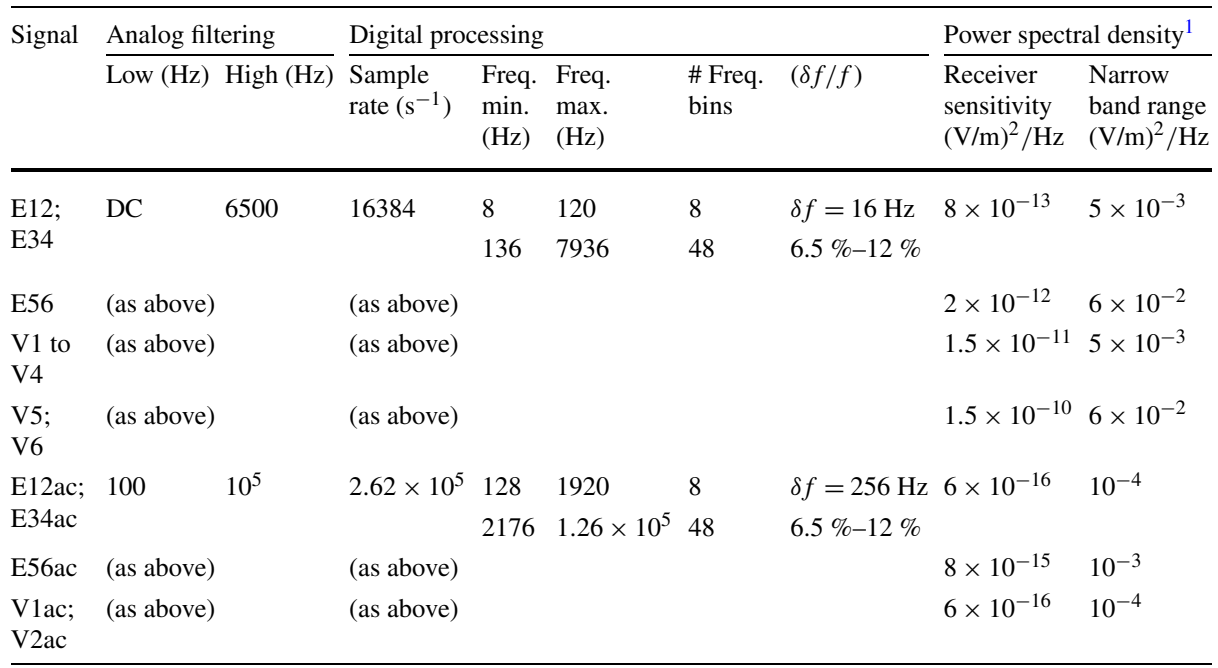

${ }^{1}$ Sensitivity of DC-coupled signals is at $1 \mathrm{kHz}$. Sensitivity of AC-coupled signals is at $10 \mathrm{kHz}$

divided into four buffers. Each buffer can hold $131072\left(2^{17}\right)$ data words (two byte words) of each of the three AC-coupled Eac or Vac signals and $32768\left(2^{15}\right)$ data words of each of the three SCM signals. At the native sampling rate of $\sim 262 \mathrm{kS} / \mathrm{s}$, the buffer holds $1 / 2$ second of continuous data from the AC-coupled signals. The HSBM is planned to operate with three signal at $\sim 65.5 \mathrm{kS} / \mathrm{s}$ holding two second intervals of AC-coupled Eac or Vac data and two second intervals of SCM data.

Of the four memory buffers, one is used as an input buffer, two are holding buffers, and one is an output buffer. The input buffer is designed as a circular memory continuously recording the data from the six signals, overwriting the oldest data. A trigger system monitors the data quality of the input buffer. If the trigger quality of the input buffer exceeds that of a holding buffer, the data in the input buffer is preserved. The input buffer becomes a holding buffer and the holding buffer with the lowest data quality becomes the input buffer.

The output buffer is used to transfer data to the MMS central burst memory. The HSBM transfer cycle is selectable at $5 \mathrm{~s}, 10 \mathrm{~s}, 20 \mathrm{~s}$, or $40 \mathrm{~s}$. After the transfer cycle is complete, the holding buffer with the highest data quality becomes the next output buffer and the output buffer becomes a holding buffer with zero data quality. In the subsolar regions, the HSBM transfer cycle is planned to be $5 \mathrm{~s}$, giving $40 \%$ coverage of the AC-coupled data at $\sim 65.5 \mathrm{kS} / \mathrm{s}$ and SCM data at $\sim 16.5 \mathrm{kS} / \mathrm{s}$. In the magnetotail, the HSBM transfer cycle is planned to be $20 \mathrm{~s}$.

The triggers nominally use the E12ac signal. They divide the buffer into 64 slices, recording the peak absolute value of the signal in each slice. The data quality value is the average of the eight highest peaks in the 64 slices.

Solitary Structure Detector The solitary structure detector is a fairly new approach to observing plasma structures such as electron phase-space holes. Its intention is to detect structures that are associated with magnetic reconnection. The solitary structure detector examines of the time series data from one of the AC-coupled channels, planned to be E12ac.

The algorithm is fairly basic. A window of data is examined periodically. The size of the window, planned to be $\sim 1 \mathrm{~ms}$ of data, can be configured. The average of the data within the 
window is calculated and subtracted from the original data in the window. The variation is estimated by averaging the absolute values all of the points in the window:

$$
\sigma_{e s t}=\langle|x(1: n)|\rangle
$$

where $x$ is a set of $n$ data values in the window. Mathematically, $\sigma_{e s t}$ differs from a true standard deviation $\sigma$. If $x$ is random noise, $\sigma_{e s t} / \sigma \sim 0.8$ whereas if $x$ is sinusoidal, $\sigma_{\text {est }} / \sigma=2 \sqrt{2} / \pi \sim 0.9$.

If the peak amplitude $\left(|x|_{\text {peak }}\right)$ exceeds the variation by a factor of four, a solitary structure is considered to be in the window. One of four counters is incremented depending on the peak value in the window, $|x|_{\text {peak }}$. The amplitude range of each of the counters can be configured. In the initial configuration, the lowest amplitude counter includes all structures with $|x|_{\text {peak }}<\sim 1 \mathrm{mV} / \mathrm{m}$. The next counters include structures with $\sim 1 \mathrm{mV} / \mathrm{m}$ $<|x|_{\text {peak }}<\sim 10 \mathrm{mV} / \mathrm{m}, \sim 10 \mathrm{mV}<|x|_{\text {peak }}<\sim 100 \mathrm{mV} / \mathrm{m}$, and $\sim 100 \mathrm{mV}<|x|_{\text {peak }}$. The counters are reported and zeroed periodically (planned once every 10 seconds) and can be used as burst triggers or as indicators of strong kinetic activity in the plasma.

It is important to note that the solitary structure detector is inexact. It was tested on data from the THEMIS satellite (e.g., Andersson et al. 2009). The solitary wave detector identified greater than $70 \%$ of electron phase-space holes and plasma structures identified by eye. It reported less than $10 \%$ false detections. Missed detections occurred in regions of extremely dense (closely-spaced in time) solitary structures. In these regions, is possible to have more than one solitary structure in the window, in which case neither is counted ( $\sigma_{e s t}$ becomes too high). False detections are statistically predictable. Even though the solitary wave detector is inexact, the results show a good correlation with electron phase-space holes; high counts rates were seen when electron phase-space holes were present and low count rates correlated with the absence of electron phase-space holes.

Data Compression and Packets The data are compressed by several methods. Waveform data undergo lossless "delta" compression. Under this algorithm, data from a specific waveform are grouped in sets of 32 words (a word is 16 bits). The first word is reported in full. The difference between the remaining 31 words is then reported with as many bits as needed so that no information is lost. The needed number of bits is reported in the second field. This type of lossless compression typically results in $25 \%-50 \%$ of the original data volume during quiet periods, with less efficient compression during active periods. The spectra undergo a pseudo-logarithmic compression to an 8-bit number representing over $140 \mathrm{~dB}$ of range at $\sim 5 \%$ accuracy. After compression, the DSP organizes the data into telemetry packets for transmission, and transfers these packets to the central instrument data processor (CIDP, see Fusilier et al. 2014, this issue) to be stored as survey or burst data and later transmitted.

The time stamp of the data packets is critical to data analysis. The DSP records the sample time of the first point in each waveform packet, which can be reconstructed to within $\sim 4 \mu \mathrm{s}$ of a spacecraft clock and $\sim 1 \mu$ s relative to other data streams in the DSP. The HSBM time stamps are the last data point in the packet.

SCM Calibration The DSP periodically creates a calibration signal for the SCM. The SCM calibration signal is a sine wave output that sweeps from a user configurable beginning frequency to a user configurable ending frequency. The sine wave dwells at each frequency for two cycles. The frequency following a dwell is 2 times the previous frequency. The beginning and ending frequencies can be configured from $0.125 \mathrm{~Hz}$ to $4096 \mathrm{~Hz}$ in factorof-two steps. The magnitude of the SCM calibration signal can be configured to $1.25 \mathrm{~V}_{\mathrm{pp}}$, $2.5 \mathrm{~V}_{\mathrm{pp}}, 5 \mathrm{~V}_{\mathrm{pp}}$, or $10 \mathrm{~V}_{\mathrm{pp}}$, where $\mathrm{V}_{\mathrm{pp}}$ is the peak-to-peak voltage. 
Since each waveform dwells for exactly 2 cycles, the gain and phase versus frequency of each SCM sensor can be determined. The signals are produced digitally by the FPGA using a CORDIC algorithm (Cully et al. 2008). The signal is fed to an 8-bit digital-to-analog converter with a 16-cycle dither creating $\sim 12$-bit accuracy.

\section{Instrument Calibration and Performance}

The ADP/AEB, SDP/BEB, and DSP are calibrated separately. The ADP sensor is calibrated with the AEB. The calibration procedure requires a well-designed environment to accurately measured the sensor, preamplifier, and AEB response in a simulated plasma environment. Comparatively, the DSP is well behaved and repeatable. It displays little variation over temperature and between channels. The calibration plan is to obtain meticulous transfer functions (phase and gain) from the ADP/AEB, from the SDP/BEB, and from the DSP, then combine the transfer functions for a final calibration. DC levels are calibrated through the entire system as a check.

\subsection{ADP Calibration and Performance}

An ADP preamplifier and AEB (Lindqvist et al. 2014, this issue) are designed to work as a single unit. There are two AEB units on each spacecraft, one for the $+\mathrm{Z}$ ADP preamplifier, the other for the $-\mathrm{Z}$ ADP preamplifier. The AEBs are mounted on the MMS spacecraft deck. The AEB supplies floating power to the preamplifier, sets the current bias to the sensor, and controls the voltages on surfaces close to the sensor (called inner guard, outer guard, and stub. The AEB also receives the signal from the preamplifier and buffers the signal before passing it to the DSP.

\subsubsection{Sensor and Plasma Coupling}

A representative calibration of an electric field instrument requires simulating the surrounding plasma. The coupling between the sensor and the surrounding plasma is often expressed as a sheath resistance $\left(R_{s h}\right)$ and a sheath capacitance $\left(C_{s h}\right)$. The sheath resistance can be estimated analytically by realizing that the sensor's DC potential $(V)$ with respect to the plasma is such that the net current to the sensor must be zero:

$$
I(V)=I_{e}(V)+I_{i}(V)+I_{p h}(V)+I_{s e c}+I_{\text {bias }}+I_{\text {other }}=0,
$$

where $I_{e}$ is the thermal electron current, $I_{i}$ is the thermal ion current, $I_{p h}$ is the photoelectron current emitted from the sensor, $I_{\text {sec }}$ is the current from secondary electrons emitted from the sensor, $I_{\text {bias }}$ is the bias current applied to the sensor, and $I_{\text {other }}$ includes stray currents such as spacecraft photoelectron currents to the sensor. In most of the plasma environments of magnetic reconnection, $I_{p h}$ and $I_{\text {bias }}$ dominate the currents, so $I_{\text {bias }}=-I_{p h}$. Since $I_{p h}$ is seen to have a exponential dependence on $V$ (Pedersen 1995):

$$
I_{p h}=I_{o} e^{-V / V_{p h}}, \quad V \geq 0
$$

where $I_{o}$ is the photoelectron current at $V=0$, and $e V_{p h}$ is the characteristic energy of the photoelectrons. The sheath resistance is estimated as:

$$
R_{s h}=\left(\frac{d I}{d V}\right)^{-1} \simeq \frac{V_{p h}}{-I_{o} e^{-V / V_{p h}}}=\frac{V_{p h}}{I_{\text {bias }}}
$$


Using nominal values, $I_{\text {bias }}=200 \mathrm{nA}$ and $V_{p h}=2.7 \mathrm{~V}, R_{s h}=13.5 \mathrm{M} \Omega$. This value is but a rough estimate as thermal electron currents are expected to reduce $R_{s h}$ and the characteristic energy of the photoelectrons is not well understood. Bias sweeps on Cluster indicate sheath resistance roughly $1 / 2$ of the value predicted above (Cully, private communication). Once on orbit, bias sweeps are planned to better determine $R_{s h}$. The ADP calibrations include resistances of $5 \mathrm{M} \Omega$ and $10 \mathrm{M} \Omega$.

In measuring plasma waves, capacitive coupling of the sensor to the plasma is critical. The free-space capacitance for a cylinder is estimated at (Jackson 2000):

$$
C_{s h} \approx \frac{2 \pi \epsilon_{o} L}{\ln (L / r)}\left[1+\frac{(1-\ln (2))}{\ln (L / r)}\right]
$$

where $L$ is the sensor length $(1 \mathrm{~m})$ and $r$ is its radius $(0.32 \mathrm{~cm}) . C_{s h}$ is calculated to be $10.2 \mathrm{pF}$ for the ADP sensors. This value can increase if the Debye length $\left(\lambda_{\mathrm{D}}\right)$ of the plasma is on the order of or smaller than $L$. Since $\lambda_{\mathrm{D}} \gg L$ in almost all plasmas MMS will study, $C_{s h}$ is not expected to vary significantly.

\subsubsection{ADP Calibration}

A true measurement of the plasma potential and the derived electric field requires that the input resistance to the preamplifier $\left(R_{i n}\right)$ is large compared to $R_{s h}$, and that the input capacitance $\left(C_{i n}\right)$ is small compared to $C_{s h}$. $C_{i n}$ includes all stray capacitances between the sensor and the spacecraft. While it is possible to design a system in which $R_{i n} \gg R_{s h}$, it is difficult to achieve $C_{i n} \ll C_{s h}$, so the transfer function (gain and phase) of the signal from the ADP must be carefully documented.

The ADP receiving elements were calibrated in a $1 \mathrm{~m} \times 1 \mathrm{~m} \times 2.5 \mathrm{~m}$ Faraday cage. The ADP RE is placed vertically in its deployed configuration in the center of the cage. In Fig. 5, one can see the tip plate and guard ring at the bottom of the cage. Just above are the stubs and hinge (silver), and the preamplifier (black). The 1-m sensor is at the top of the RE. A wire from the cage to the top of the receiving element is terminated at the end of the sensor with a resistor to simulate the plasma. This "drive resistor" had a range of values, depending on the specific test. The cage and resistor are the sole stimulation of the RE sensor.

The calibration process included measurements of the input impedance, DC gain, offsets, bias currents, and inner guard, outer guard, and stub voltages. The input impedance is determined using a $1 \mathrm{G} \Omega$ as the driving resistor. All eight of the flight systems recorded an input impedance of greater than the goal, $R_{i n}>2 \times 10^{11} \Omega$, with most having $R_{i n}>10^{12} \Omega$. Clearly $R_{i n} \gg R_{s h}$, so the DC gains are very close to unity.

The transfer function, or gain and phase versus frequency, is recorded using several values of resistance. Figures $6 \mathrm{a}$ and $6 \mathrm{~b}$ show the gain and phase response of the $+\mathrm{Z}$ and $-\mathrm{Z}$ pair of REs on one of the MMS satellites. The gain response remains is nearly unity in the frequency range from $\mathrm{DC}$ to $100 \mathrm{~Hz}$ where resistive coupling dominates. From $\sim 100 \mathrm{~Hz}$ to $\sim 3 \mathrm{kHz}$, the floating power supply bootstrapping minimizes the gain loss from stray capacitance. For frequencies above $\sim 10 \mathrm{kHz}$, the gain is roughly 0.5 indicating that $C_{i n} \sim C_{s h}$. It is important to note that the ADP preamplifier response was repeatable from unit to unit to within $1 \%$ in gain and phase.

Figure 7 displays an electrical model of the ADP preamplifier, cable $(\sim 15 \mathrm{~m})$, and AEB. The model consists of four elements: (1) an input network, (2) a low-pass filter, (3) a cable delay, and (4) a summing network. The input network includes $R_{s h}$ and $C_{s h}$ to represent the sensor coupling to the plasma. $C_{i n}$ is a critical part of the input network, but $R_{i n}$ is 
Fig. 5 The ADP RE inside of the Faraday cage during calibration

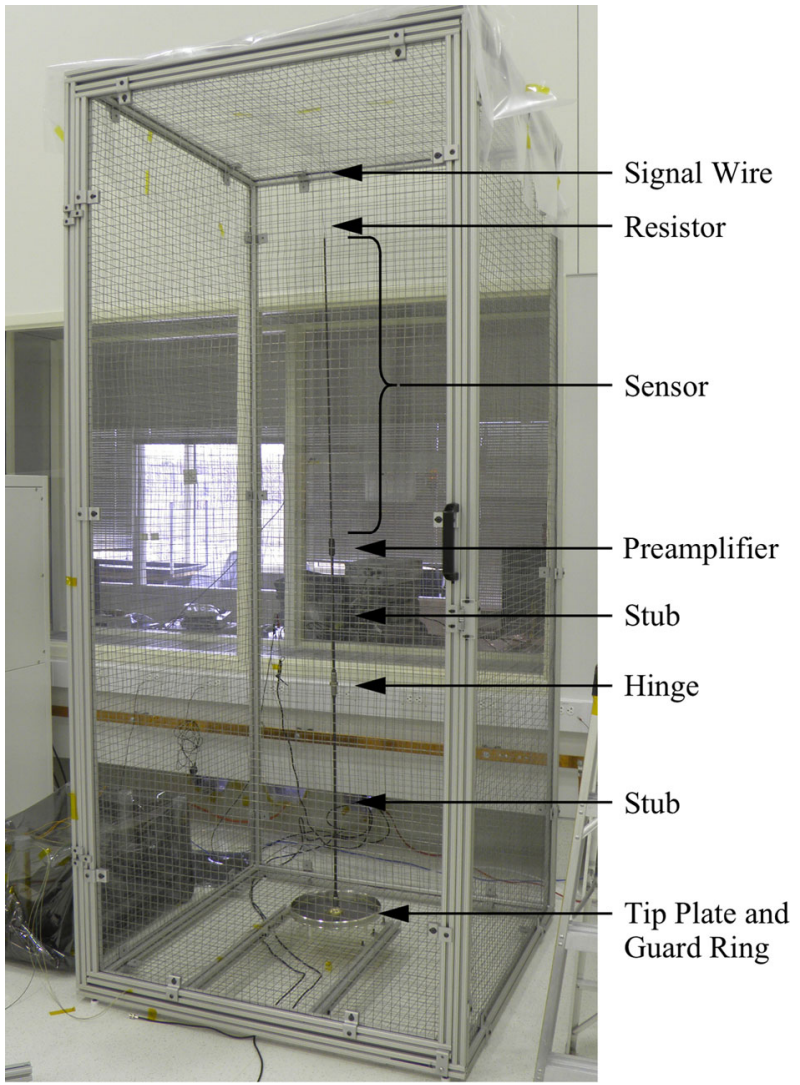

sufficiently large that it is not required. The low-pass filter and delay networks simulate the preamplifier and $15-\mathrm{m}$ cable between the preamplifier and the AEB. The summing network is required to simulate the response between $\sim 100 \mathrm{kHz}$ and $3 \mathrm{kHz}$ where the AEB's floating power supply, guard, and stub bootstrapping have an effect.

The network model was fit to the ADP calibrations. In performing the fit, $C_{s h}, C_{i n}, f_{c b l}$, $f_{1}, f_{2}$, and $\tau_{D}$ are varied (under limits) to obtain the best result. The red lines in Fig. 6 shows the best fit to the preamplifier response in gain (top panel) and phase (bottom panel). These fits indicate that the network model, while simplified, represents the preamplifier response well. Developing such a model is critical since it allows for digital correction to the measured electric field signal. Without such correction, for example, a bipolar electron phase-space hole may appear to be a tri-polar structure due to the distortion of the complex transfer function of the preamplifier and AEB system.

The fits revealed $C_{s h}$, which in this case is the capacitance coupling between the Faraday cage and sensor, to be $12.0 \pm 0.1 \mathrm{pF}$. Through analytical estimates, the Faraday cage was expected to have $\sim 11.5 \mathrm{pF}$ coupling. The remaining $\sim 0.5 \mathrm{pF}$ comes from the $5 \mathrm{M} \Omega$ resistor driving the sensor. This calibration and analysis indicates that the free-space value of $C_{s h}$, $10.2 \mathrm{pF}$, is accurate to within a few percent except in dense plasmas. $C_{i n}$ is determined to be $10.5 \pm 0.1 \mathrm{pF}$ for the above fit. This value varies up to $0.2 \mathrm{pF}$ from unit to unit. The input characteristics of all ADP preamplifiers are documented. 
Fig. 6 (Top) The gain versus frequency of the $+\mathrm{Z}$ (circles) and $-\mathrm{Z}$ (crosses) ADP with a drive resistor of $5 \mathrm{M} \Omega$. The red line is a derived analytic transfer function. (Bottom) The phase versus frequency
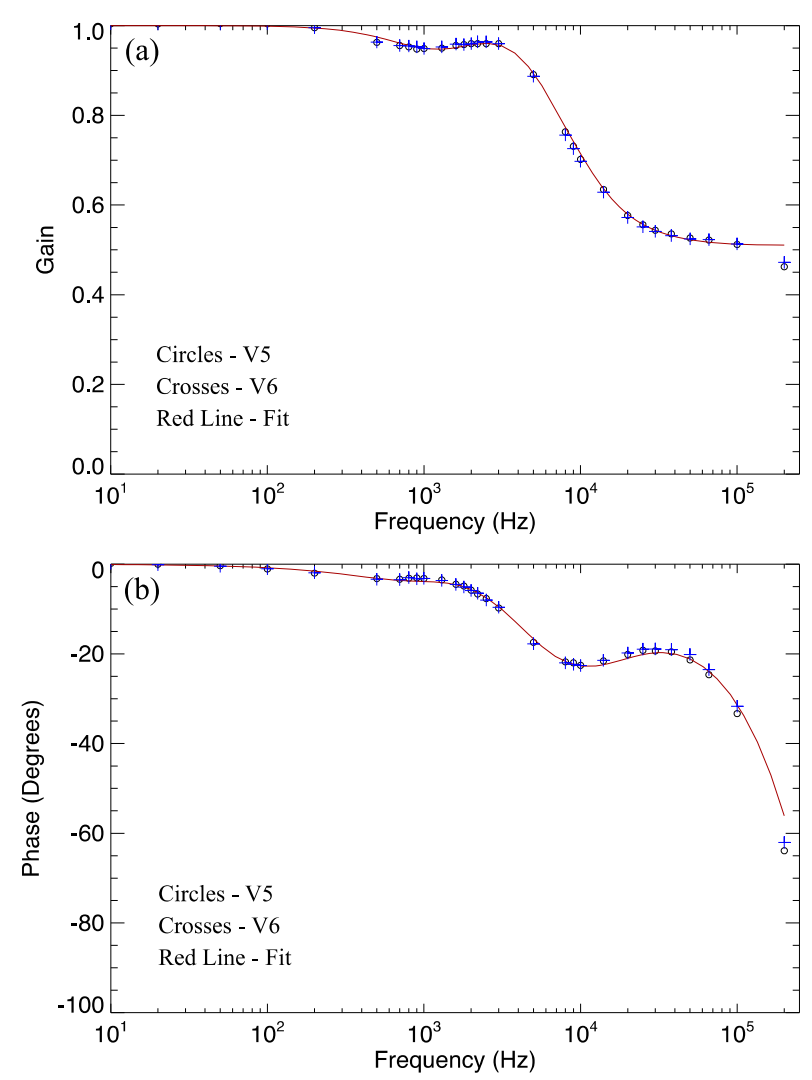

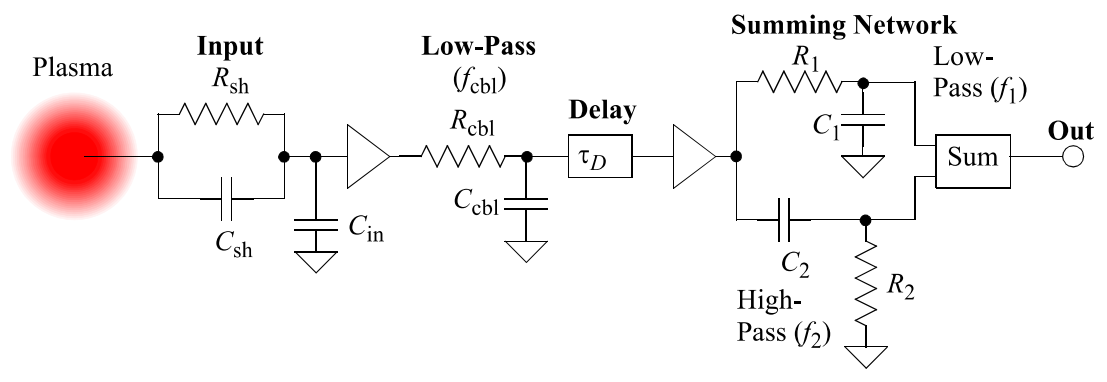

Fig. 7 A network equivalent to model the ADP Preamp. The input network models the plasma and sensor. The low-pass and delay simulate the preamplifier and 15-m cable to the AEB. The summing network corrects for AEB bootstrapping of the floating power supply, stub, and guards

The fits also reveal a high-pass pole $f_{c b l} \sim 200 \mathrm{kHz}$ and the delay time, $\tau_{D} \sim 0.5 \mu \mathrm{s}$. These values are within expected transmission delays and delays within the amplifier. The summing network varies between AEB channels. Its effect corrects the gain by $<5 \%$ and adds very little distortion to the signal. In separate tests (not shown), the model network closely predicted the step function response of the ADP preamplifier. Furthermore, it was shown that the output signal could be digitally corrected so that it has no visible distortion. 


\subsection{DSP Calibration and Performance}

DSP calibrations primarily are from stimulating the DSP at its inputs and comparing the output to the stimulation signals. The DSP was verified to have better than $\sim-80 \mathrm{~dB}$ common mode rejection in the construction of E signals at DC. It also is shown to have better than $-80 \mathrm{~dB}$ (except in one case, which was $-78 \mathrm{~dB}$ ) cross talk between E and SCM channels and DC gains are known and stable to $\sim 0.02 \%$. The noise levels of each channels is recorded (see Tables 3 and 5) and the dynamic ranges and frequency ranges are within design specifications. In general, the distortions and errors from DSP processing (except digitization and saturation) are negligible when compared to those of the sensors.

The E, V, and SCM signals are all treated with identical low-pass, anti-aliasing filters with constant time delay (minimum signal distortion). Each of these filters has been characterized and the differences have been recorded. As discussed above, the time delays in the DSP signal processing can be significant. These delays, which are repeatable, have been tabulated.

The DSP flexibility, while scientifically valuable, creates a great challenge in digital testing and calibration. Each of the science signals has a number of configurations and data rates. For example, E12 (DC-coupled) can be set to any of 16 data rates. The spectral averaging can be adjusted and the broadband filters can have adjustable output rates and/or can be set to peak, average, or both. Including all signals (and analog-generated signals), the DSP basically has $2^{\mathrm{N}}$ configurations, where $\mathrm{N}$ is on the order of 1000 , making the DSP impossible to fully test. Since digital processing is identical on all of signals, the effort concentrated on (1) calibration of individual channels at their native rates, (2) calibration of the planned in-flight configurations, and (3) automated verification of each of the signals at all of their configurations while the other signals remain in a nominal configuration.

\section{ADP and DSP Operations and In-Flight Calibration}

The ADP sensors require in-flight calibration to achieve the needed accuracy. Unlike the SDP, the ADP signals are not modulated by the spacecraft spin, so DC offsets are difficult to remove. The ADP bias current and stub and guard voltages need to be adjusted for differing plasma environments to minimized signal errors. When present, the electric field generated by the spacecraft wake (Eriksson et al. 2006) needs to be identified manually.

The DSP requires little on-orbit calibration or maintenance. However, its flexibility makes it useful to optimize scientific return, so the planned DSP configurations may be changed during the mission to focus on new discoveries or overcome sensor problems.

\subsection{ADP Bias Currents and Stub and Guard Voltages}

After deployment, the ADP bias currents, guard voltages, and stub voltages are optimized. Past experience (Mozer, private communication) indicates that the photoelectron yield of DAG213 surfaces increases over a period of several months immediately after launch. Exposure to atomic oxygen can rapidly lower the photoelectron yield. MMS is expected to experience some atomic oxygen exposure early in the mission before achieving its final orbit. Fortunately, the MMS spacecraft have a high enough perigee that atomic oxygen exposure is expected to be negligible after the initial orbital maneuvers.

During the first few months on orbit, the commissioning phase, the ADP RE will be run though a series of bias sweeps. These sweeps involve stepping the bias current though 
the full range at moderate resolution (see Lindqvist et al. 2014, this issue for details). From these bias current sweeps, one can determine the photoelectron yield and estimate $R_{s h}$ as a function of bias current. Bias currents will be adjusted to minimize differences between the $+\mathrm{Z}$ and $-\mathrm{Z}$ sensors and achieve as low value on $R_{s h}$ as possible.

The stub voltages on the ADP also are expected to have an influence on the ADP performance. The stub sections are bootstrapped to follow the ADP output in the frequency range from $\mathrm{DC}$ to $\sim 300 \mathrm{~Hz}$. The stub is initially adjusted to a small positive offset $(\sim 1 \mathrm{~V})$ from the ADP sensor to reduce the photoelectron current from the stub to the sensor. Two types of sweeps are planned for the stubs. A course sweep will engage the entire offset range $( \pm 10 \mathrm{~V})$. After narrowing down the best operating range, a fine sweep will be used to determine the offset. The guard voltages have a lower-order influence on the ADP performance. A two-step sweep (coarse then fine) is also planned for the inner and out guards. Initially, the outer guard is set to $+5 \mathrm{~V}$ to attract photoelectrons, whereas the inner guard is set to zero.

The commissioning phase is expected to take several months during which the bias currents, stub voltages, and guard settings are optimized primarily in tenuous plasmas. During routine operations, the ADPs will undergo regular set of bias sweeps two times an orbit to maintain optimized performance in a variety of plasma conditions.

\subsection{ADP In-Flight Calibration}

Two methods are employed for ADP in-flight calibration. Torbert et al. (2014, this issue) describe this process, so we provide a brief description. The first method is to directly compare the ADP measurements with those from the electron drift instrument. The electron drift instrument is known to have high accuracy $(\sim 0.1 \mathrm{mV} / \mathrm{m})$ on DC electric field in periods of low activity (low variation in electric field strength) and moderate magnetic field amplitude. Since ADP offsets and effective antenna length vary slowly, the offset and gains corrections derived by this processes can be employed through periods when data from the electron drift instrument are not available.

The second method compares $\mathbf{E}$ signals with the $\boldsymbol{u} \times \boldsymbol{B}$ signal over long periods to determine the effective antenna length. Over many orbits, the expected electric filed signal along the axial boom can be estimated from previous satellite measurements (it is nearly zero). The velocity vector of the spacecraft $(\mathbf{u})$ and the magnetic field $\left(\mathbf{B}_{D C}\right)$ are well measured and dominate the long-term average of the ADP electric field. Correlation of ADP measurements with the $\boldsymbol{u} \times \boldsymbol{B}$ signal over long periods allows for adjustment of the effective antenna length.

While parallel electric fields are a key element of the MMS measurements, they are not expected to a have significant amplitude when averaged over periods of tens of seconds. Combining SDP, ADP, and magnetometer data, one can test if $\boldsymbol{E} \cdot \boldsymbol{B}_{D C}=0$ over moderate periods (tens of seconds). When the ADP and SDP both measure part of the parallel electric field, the DC offset on the ADP can be determined and removed. This process proved successful and the FAST, Polar, and THEMIS missions.

\section{Summary}

The ADP and DSP, when combined with the SDP, BEB, AEB, and central electronics board (Torbert et al. 2014, this issue), complete the electric field double probe instrument. The double probe instrument measures the DC to $\sim 100 \mathrm{kHz}$ electric field to an expected accuracy of better than $0.5 \mathrm{mV} / \mathrm{m}$ in the spin plane and $\sim 1 \mathrm{mV} / \mathrm{m}$ along the spin axis in 
most of the plasma environments MMS is expected to encounter. The ADPs are over $30 \mathrm{~m}$ tip-to-tip, the longest baseline on an axial DC electric field ever attempted in space. The ADPs are designed so that the spacecraft potential field acts equally on the two ADP sensors and that photoelectron currents do not vary with spacecraft spin. The DSP units on the MMS spacecraft accept nine signals from the ADP, SDP, and SCM. They are designed to perform precision analog conditioning with excellent common-mode rejection and very low crosstalk and have 16-bit A/D conversion with rates up to $\sim 262 \mathrm{kS} / \mathrm{s}$. Digital processing includes digital filters, spectral processing, high-speed burst memory, solitary structure detection, and data compression. The DSP designed to maximize maximum scientific return under a limited telemetry volume.

Open Access This article is distributed under the terms of the Creative Commons Attribution License which permits any use, distribution, and reproduction in any medium, provided the original author(s) and the source are credited.

\section{References}

L. Andersson et al., New features of electron phase space holes observed by the THEMIS mission. Phys. Rev. Lett. 102(22), 225004 (2009)

Baker et al., MMS data management. Space Sci. Rev. (2014, this issue)

J.W. Bonnell, F.S. Mozer, G.T. Delory, A.J. Hull, R.E. Ergun, C.M. Cully, V. Angelopoulos, P.R. Harvey, The electric field instrument (EFI) for THEMIS. Space Sci. Rev. 141, 303-341 (2008). doi:10.1007/ s11214-008-9469-2

J.L. Burch, J.F. Drake, Reconnecting magnetic fields. Am. Sci. 97, 392-399 (2009)

J.L. Burch et al. Magnetospheric multiscale overview and science objectives. Space Sci. Rev. (2014, this issue)

C.M. Cully, R.E. Ergun, K. Stevens, A. Nammari, J. Westfall, The THEMIS digital fields board. Space Sci. Rev. 141, 343-355 (2008)

C.M. Cully, R.E. Ergun, A.I. Eriksson, Electrostatic structure around spacecraft in tenuous plasma. J. Geophys. Res. 112, A09211 (2007). doi:10.1029/2007JA012269

J.F. Drake, M. Swisdak, C. Cattell, M.A. Shay, B.N. Rogers, A. Zeiler, Formation of electron holes and particle energization during magnetic reconnection. Science 299, 873-877 (2003). doi:10.1126/ science. 1080333

A.I. Eriksson, M. André, B. Klecker, H. Laakso, P.-A. Lindqvist, F. Mozer, G. Paschmann, A. Pedersen, J. Quinn, R. Torbert, K. Torkar, H. Vaith, Electric field measurements on Cluster: comparing the doubleprobe and electron drift techniques. Ann. Geophys. 24, 275 (2006)

R.E. Ergun et al., The FAST satellite field instrument. Space Sci. Rev. 98(1/2), 67-91 (2001)

R.E. Ergun et al., Observations of double layers in Earth's plasma sheet. Phys. Rev. Lett. 102(15), 155002 (2009). doi:10.1103/PhysRevLett.102.155002

S.A. Fusilier et al., Magnetospheric multiscale science mission profile and operations. Space Sci. Rev. (2014, this issue). doi:10.1007/s11214-014-0087-X

M. Hesse, N. Aunai, J. Birn, P. Cassak, R.E. Denton, J.F. Drake, T. Gombosi, M. Hoshino, W. Matthaeus, D. Sibeck, S. Zenitani, Theory and modeling for the magnetospheric multiscale mission. Space Sci. Rev. (2014, this issue). doi:10.1007/s11214-014-0078-y

J.D. Jackson, Charge density on thin straight wire, revisited. Am. J. Phys. 68, 789 (2000). doi:10.1119/1. 1302908

G. Lapenta, S. Markidis, A. Divin, M.V. Goldman, D.L. Newman, Bipolar electric field signatures of reconnection separatrices for a hydrogen plasma at realistic guide fields. Geophys. Res. Lett. 38, L17104 (2011). doi:10.1029/2011GL048572

O. Le Contel et al., The search-coil magnetometer for MMS. Space Sci. Rev. (2014, this issue). doi:10. 1007/s11214-014-0096-9

P.-A. Lindqvist et al., The spin-plane double probe instrument for MMS. Space Sci. Rev. (2014, this issue). doi:10.1007/s11214-014-0116-9

D. Pankow, R. Besuner, R. Wilkes, R. Ullrich, Deployment mechanisms on the fast satellite: magnetometer, radial wire, and axial booms. Space Sci. Rev. 98(1/2), 93-111 (2001)

A. Pedersen, Solar wind and magnetosphere plasma diagnostics by spacecraft electrostatic potential measurements. Ann. Geophys. 13, 118 (1995)

R.B. Torbert et al., The FIELDS instrument suite on MMS: scientific objectives, measurements, and data products. Space Sci. Rev. (2014, this issue). doi:10.1007/s11214-014-0109-8 